\title{
ESTABELECIMENTO DE CONDIÇÕES PARA ENSAIO DE DISSOLUÇÃO DE CÁPSULAS DE CINARIZINA EMPREGANDO PLANEJAMENTO FATORIAL
}

Patrícia Alves Franco Gehring, Olímpia Maria Martins Santos, Gislaine Ribeiro Pereira e Magali Benjamim de Araújo

Faculdade de Ciências Farmacêuticas, Universidade Federal de Alfenas, Rua Gabriel Monteiro da Silva, 700, 37130-000 Alfenas - MG, Brasil

\section{Rudy Bonfilio*}

Departamento de Fármacos e Medicamentos, Faculdade de Ciências Farmacêuticas, Universidade Estadual Paulista, Rod. AraraquaraJaú, km 1, 14801-902 Araraquara - SP, Brasil

Recebido em 5/7/10; aceito em 21/10/10; publicado na web em 26/1/11

\begin{abstract}
ESTABLISHMENT OF DISSOLUTION TEST CONDITIONS FOR CINNARIZINE IN PHARMACEUTICAL CAPSULES USING FACTORIAL DESIGN. This work describes the establishment of dissolution test conditions for $75 \mathrm{mg}$ cinnarizine capsules using a multivariate approach. A $2^{3}$ full factorial design was carried out to achieve the best conditions and $\mathrm{HCl} 0.1 \mathrm{~mol} \mathrm{~L}^{-1}$ as dissolution medium, basket as apparatus at $100 \mathrm{rpm}$ and collect time at $30 \mathrm{~min}$ were considered adequate. The quantification was carried out by spectrophotometry at $251 \mathrm{~nm}$. Both dissolution procedure and analytical method were validated and all parameters were within the acceptable limits. Since there is no official monograph for this pharmaceutical product, this dissolution test could be applied for quality control routine.
\end{abstract}

Keywords: dissolution test; cinnarizine; $2^{3}$ full factorial design.

\section{INTRODUÇÃO}

Os ensaios de dissolução in vitro para formas farmacêuticas orais de liberação imediata são de fundamental importância, pois são utilizados para garantir a qualidade lote a lote, orientar o desenvolvimento de novas formulações e assegurar a uniformidade da qualidade e do desempenho do medicamento após determinadas alterações. ${ }^{1}$ Desse modo, torna-se imperativo realizar estudos de dissolução in vitro, a fim de assegurar a liberação da substância ativa da forma farmacêutica, garantindo assim sua qualidade biofarmacêutica..$^{2-4}$

Dentre os produtos de liberação imediata, a cinarizina (Figura 1) tem sido amplamente manipulada no mercado brasileiro na forma de cápsulas. O fármaco é um vasodilatador periférico e cerebral, utilizado no tratamento de transtornos isquêmicos, encefálicos ou periféricos, particularmente em síndromes labirínticas..$^{5-7}$<smiles>C(=C/c1ccccc1)\CN1CCN(C(c2ccccc2)c2ccccc2)CC1</smiles>

Figura 1. Estrutura química da cinarizina

Uma revisão na literatura revela que existem inúmeros trabalhos sobre métodos analíticos para determinação de cinarizina em formulações farmacêuticas. Os métodos descritos empregam as técnicas de eletroforese capilar, ${ }^{8}$ eletrocromatografia capilar, ${ }^{9}$ cromatografia gasosa, ${ }^{10,11}$ cromatografia líquida de alta eficiência, ${ }^{12-19}$ cromatografia em camada delgada, ${ }^{18}$ cromatografia em camada delgada de alta efi-

*e-mail: rudybonfilio@yahoo.com.br ciência, ${ }^{20,21}$ métodos eletroquímicos, ${ }^{22-26}$ espectrofotometria,,${ }^{16,17,19,26-41}$ quimioluminescência, ${ }^{42}$ fluorimetria ${ }^{43,44}$ e espectrodensitometria. ${ }^{19}$ Além dos métodos mencionados acima, ensaios de dissolução para comprimidos de cinarizina utilizando detecção potenciométrica ou por cromatografia líquida de alta eficiência também são descritos. ${ }^{45,46}$

Até o presente momento, porém, não se encontra descrito na literatura um ensaio de dissolução para cápsulas de cinarizina e nenhuma farmacopeia descreve uma monografia para esta forma farmacêutica. Considerando a importância dos ensaios de dissolução in vitro para formas farmacêuticas orais de liberação imediata e a grande frequência de prescrição de cápsulas de cinarizina no mercado brasileiro, o presente trabalho objetivou o estabelecimento e a padronização de um ensaio de dissolução de cápsulas manipuladas contendo $75 \mathrm{mg}$ de cinarizina, utilizando uma abordagem multivariada.

A abordagem multivariada propicia uma redução no número de experimentos, é mais fácil de implementar e apresenta melhor relação custo-benefício em relação à abordagem univariada. ${ }^{47}$ Portanto, um planejamento fatorial completo mostrou-se adequado aos propósitos desse trabalho.

\section{PARTE EXPERIMENTAL}

Produtos farmacêuticos, padrão de referência e reagentes

Foram analisadas cápsulas de cinarizina $75 \mathrm{mg}$ manipuladas por três diferentes farmácias, denominadas formulações A, B e C, respectivamente. Segundo os fornecedores, as cápsulas analisadas continham lauril sulfato de sódio, estearato de magnésio, aerosil, talco e amido, além do princípio ativo. A composição quantitativa dos excipientes não foi informada. Utilizou-se como padrão de trabalho cinarizina base livre (Galena ${ }^{\circledR}$, Campinas, Brasil), com teor declarado de $99,8 \%$. Para o ensaio de dissolução utilizou-se ácido clorídrico grau analítico (Vetec ${ }^{\circledR}$, Rio de Janeiro, Brasil). 


\section{Equipamentos}

Foram utilizados os seguintes equipamentos: aparelho de dissolução Electrolab ${ }^{\circledR}$, modelo TDT-08L, equipado com 8 cubas (Mumbai, Índia); espectrofotômetro UV/Vis Shimadzu ${ }^{\circledR}$, modelo UV-1601 PC (Kyoto, Japão); balança analítica Kern ${ }^{\circledR}$, modelo 410 (Kern, Alemanha); medidor de pH Marconi ${ }^{\circledR}$, modelo PA 200 (Piracicaba, Brasil); sistema de purificação de água TKA modelo LAB - UPW (Jena, Alemanha); sistema de filtração a vácuo Millipore ${ }^{\circledR}$ (Bedford, EUA); aparelho de ultrassom Unique ${ }^{\circledR}$, modelo USC1400 (São Paulo, Brasil); bomba de vácuo Nova Técnica ${ }^{\circledR}$ (Piracicaba, Brasil).

\section{Preparo de soluções}

O meio de dissolução solução de $\mathrm{HCl} 0,1 \mathrm{~mol} \mathrm{~L}^{-1}$ foi preparado dissolvendo-se $8,5 \mathrm{~mL}$ de ácido clorídrico em $1 \mathrm{~L}$ de água purificada. O meio de dissolução, em todos os experimentos, foi previamente desaerado à $41^{\circ} \mathrm{C}$ em banho de ultrassom por $30 \mathrm{~min}$.

A solução padrão de cinarizina foi preparada pesando-se exatamente $20,83 \mathrm{mg}$ de cinarizina padrão secundário, que foi transferida quantitativamente para balão volumétrico de $100 \mathrm{~mL}$. Adicionaram-se cerca de $50 \mathrm{~mL}$ de solvente e deixou-se em banho de ultrassom por 20 min, completando-se o volume do balão com o mesmo solvente. Soluções padrão de trabalho foram preparadas imediatamente antes do uso, através de adequadas diluições da solução-mãe padrão de cinarizina, utilizando solução de $\mathrm{HCl} 0,1 \mathrm{~mol} \mathrm{~L}^{-1}$ como solvente.

As soluções das amostras foram preparadas transferindo-se uma cápsula para cada cuba contendo $900 \mathrm{~mL}$ de meio de dissolução (solução de $\mathrm{HCl} 0,1 \mathrm{~mol} \mathrm{~L}^{-1}$ ) na temperatura de $37 \pm 0,5^{\circ} \mathrm{C}$. As amostras foram coletadas no tempo especificado em cada experimento, imediatamente filtradas em papel de filtro quantitativo $\operatorname{Vetec}^{\circledR}$ e diluídas 10 vezes para posterior análise por espectrofotometria.

\section{Medidas espectrofotométricas}

Varreduras espectrais nas faixas de absorção entre 400 e 200 $\mathrm{nm}$ foram realizadas para identificação do pico de máxima absorção da cinarizina, usando cubetas de quartzo de $1 \mathrm{~cm}$, velocidade de varredura rápida, intervalo de agrupamento de dados de $2,0 \mathrm{~nm}$ e abertura de fenda de $2,0 \mathrm{~nm}$. As concentrações do fármaco no meio de dissolução foram determinadas através de medidas de absorvância no comprimento de onda de $251 \mathrm{~nm}$.

\section{Planejamento fatorial}

Para se estabelecer as condições adequadas à realização de ensaio de dissolução de cápsulas de cinarizina, utilizou-se um planejamento fatorial completo $2^{3} .^{48}$ Neste tipo de planejamento, os fatores (variáveis) que se deseja estudar são analisados em dois níveis, um nível alto, codificado pelo sinal positivo (+) e um nível baixo, codificado pelo sinal negativo (-). Neste trabalho, os fatores analisados foram o aparato (cesta e pá), velocidade de agitação (50 e 100 rpm) e tempo de ensaio para realização de coleta de amostras (30 e $60 \mathrm{~min}$ ). A Tabela 1 mostra as codificações estabelecidas para cada fator na construção do planejamento fatorial completo $2^{3}$.

Após definição dos fatores e níveis a serem empregados, construiu-se uma matriz de planejamento, levando-se em consideração todas as possíveis combinações entre os níveis dos fatores. Para um experimento completo do tipo $2^{3}$ foram realizados 8 experimentos, os quais estão demonstrados na Tabela 2.

Os ensaios de dissolução foram realizados em duplicata e em ordem aleatória. As amostras foram preparadas transferindo-se uma cápsula para cada cuba contendo $900 \mathrm{~mL}$ de meio de dissolução (solução
Tabela 1. Fatores e níveis estabelecidos para construção de planejamento fatorial completo $2^{3}$

\begin{tabular}{ccc}
\hline Fatores & \multicolumn{2}{c}{ Níveis } \\
\cline { 2 - 3 } & (-) Baixo & (+) Alto \\
\hline Aparato (F1) & Pá & Cesta \\
Velocidade (F2) & $50 \mathrm{rpm}$ & $100 \mathrm{rpm}$ \\
Tempo (F3) & $30 \mathrm{~min}$ & $60 \mathrm{~min}$ \\
\hline
\end{tabular}

Tabela 2. Matriz de planejamento fatorial completo $2^{3}$ para definição de condições de dissolução para cápsulas de cinarizina

\begin{tabular}{cccc}
\hline Ensaio & $\begin{array}{c}\text { F1 } \\
\text { Aparato }\end{array}$ & $\begin{array}{c}\text { F2 } \\
\text { Velocidade }\end{array}$ & $\begin{array}{c}\text { F3 } \\
\text { Tempo }\end{array}$ \\
& $\begin{array}{c}-(\text { Pá }) \\
+(\text { Cesta })\end{array}$ & $\begin{array}{c}-(50 \mathrm{rpm}) \\
-(100 \mathrm{rpm})\end{array}$ & $+(60 \mathrm{~min})$ \\
& - & - & - \\
\hline 1 & + & - & - \\
2 & - & + & - \\
3 & + & + & - \\
4 & - & - & + \\
5 & + & - & + \\
6 & - & + & + \\
7 & + & + & + \\
8 & & &
\end{tabular}

de $\left.\mathrm{HCl} 0,1 \mathrm{~mol} \mathrm{~L}^{-1}\right)$ à temperatura de $37 \pm 0,5^{\circ} \mathrm{C}$. Após a realização de cada ensaio, alíquotas foram coletadas do meio, filtradas em papel de filtro quantitativo e diluídas 10 vezes em meio de dissolução. As concentrações do fármaco no meio de dissolução foram determinadas empregando-se espectrofotometria na região do ultravioleta em 251 $\mathrm{nm}$. As respostas analíticas obtidas foram expressas em porcentagem de dissolução (\%) e foram obtidas através de comparação entre as absorvâncias de cada amostra e a absorvância de solução padrão em mesmo solvente, na concentração de $8,33 \mu \mathrm{g} \mathrm{mL} \mathrm{m}^{-1}$.

Os valores de porcentagem de dissolução obtidos em cada experimento foram então utilizados para construção de um diagrama de Pareto, que é um recurso gráfico obtido através do software Statistica, versão 6.0 (StatSoft, Inc., Tulsa, EUA). O diagrama de Pareto ordena os efeitos das variáveis e calcula quais efeitos são estatisticamente significativos, ou seja, quais variáveis influenciam significativamente na resposta analítica (porcentagem de dissolução de cinarizina). Além disso, deve-se considerar que efeitos positivos indicam que maiores níveis para cada fator produzem uma maior liberação de cinarizina nos meios de dissolução e efeitos negativos indicam que os menores níveis de determinado fator tendem a produzir uma maior resposta analítica (porcentagem de liberação de cinarizina).

\section{Perfis de dissolução}

A partir das condições otimizadas obtidas através da realização do planejamento fatorial, foram testadas as variáveis estabelecidas, realizando-se os perfis de dissolução das formulações A, B e C. Para a construção de cada perfil, 6 cápsulas foram adicionadas individualmente em cubas contendo $900 \mathrm{~mL}$ de meio de solução de $\mathrm{HCl} 0,1$ mol L ${ }^{-1}$ à temperatura de $37 \pm 0,5{ }^{\circ} \mathrm{C}$. Retiraram-se alíquotas nos tempos de 15, 20, 25, 30, 35, 40, 45, 50, 55 e $60 \mathrm{~min}$, repondo-se o mesmo volume de meio a $37 \pm 0,5^{\circ} \mathrm{C}$. As alíquotas coletadas foram filtradas em papel de filtro quantitativo e diluídas 10 vezes em meio de dissolução. As concentrações do fármaco no meio de dissolução foram determinadas empregando-se espectrofotometria na região do 
ultravioleta em $251 \mathrm{~nm}$. As respostas analíticas obtidas foram expressas em porcentagem de dissolução (\%) e foram obtidas através de comparação entre as absorvâncias de cada amostra e a absorvância de solução padrão em mesmo solvente, na concentração de $8,33 \mu \mathrm{g} \mathrm{mL} \mathrm{L}^{-1}$.

\section{Validação do ensaio de dissolução e do método de quantificação}

A validação do ensaio de dissolução e do método de quantificação foi embasada em critérios estabelecidos pela Resolução RE $n^{\circ}$ 899, de 29/5/2003, da Agência Nacional de Vigilância Sanitária, ${ }^{49}$ Official Methods of Analysis of AOAC International, ${ }^{50}$ International Conference on Harmonization $(\mathrm{ICH})^{51} \mathrm{e}$ na literatura, ${ }^{52}$ através da determinação dos parâmetros de seletividade, linearidade, precisão, exatidão, estabilidade em solução, robustez, limite de detecção e limite de quantificação.

\section{Seletividade (procedimento de dissolução)}

Para avaliação da seletividade do método analítico empregado ao procedimento de dissolução, placebos correspondentes ao processo de manipulação das cápsulas das formulações A, B e C foram submetidos a um ensaio de dissolução utilizando cesta na velocidade de agitação de $150 \mathrm{rpm}$. Após $1 \mathrm{~h}$, as amostras foram filtradas e avaliadas através de varredura espectral entre 400 e $200 \mathrm{~nm}$. Os espectros foram comparados com espectro obtido de solução de cinarizina padrão secundário na concentração de $8,3 \mu \mathrm{g} \mathrm{mL} \mathrm{e}^{-1} \mathrm{em}$ solução de $\mathrm{HCl} 0,1 \mathrm{~mol} \mathrm{~L}^{-1}$.

\section{Linearidade}

Foi estabelecida através da construção da curva analítica obtida com soluções de cinarizina padrão secundário nas concentrações de 6,$8 ; 7,6 ; 8,4 ; 9,2$ e $10,8 \mu \mathrm{g} \mathrm{mL}^{-1}$, preparadas em solução de $\mathrm{HCl} 0,1$ mol L $\mathrm{L}^{-1}$ e quantificadas pelo método espectrofotométrico na região do ultravioleta em $251 \mathrm{~nm}$. Foram preparadas 5 réplicas de cada concentração. A análise de regressão linear foi realizada através do método dos mínimos quadrados.

\section{Precisão (método de análise)}

Para avaliação da precisão do método de análise, utilizaram-se 6 pesagens de $75 \mathrm{mg}$ de padrão de cinarizina, que foram adicionados individualmente em 6 cubas do dissolutor no mesmo dia (intradia) e em um segundo dia (interdia), procedendo-se ao ensaio de dissolução nas condições padronizadas. Alíquotas foram coletadas e diluídas 10 vezes em meio de dissolução. Procedeu-se à leitura em espectrofotômetro no comprimento de onda de $251 \mathrm{~nm}$ e as médias das absorvâncias e foram calculadas as estimativas dos desvios padrões relativos (DPR) entre as determinações.

\section{Precisão (procedimento de dissolução)}

Seis cápsulas de cada formulação A, B e C foram submetidas ao ensaio de dissolução no mesmo dia (intradia) e em um segundo dia (interdia) nas condições padronizadas para o ensaio de dissolução. Após a realização dos ensaios, alíquotas foram coletadas, filtradas em papel de filtro quantitativo e diluídas 10 vezes em meio de dissolução. As concentrações do fármaco no meio de dissolução foram determinadas empregando-se espectrofotometria na região do ultravioleta em 251 $\mathrm{nm}$. As respostas analíticas obtidas foram expressas em porcentagem de dissolução (\%) e foram obtidas através de comparação entre as absorvâncias de cada amostra e a absorvância de solução padrão em mesmo solvente, na concentração de $8,33 \mu \mathrm{g} \mathrm{mL}^{-1}$. Foram calculadas as médias das determinações e a estimativa dos DPR.

\section{Exatidão (método de análise)}

A exatidão do método de análise foi avaliada através da adição em balões volumétricos de quantidades conhecidas de padrão se- cundário aos placebos das cápsulas das formulações A, B e C, para obtenção em triplicata de três níveis de concentração da curva de calibração: 6,8; 8,4 e 10,8 $\mu \mathrm{g} \mathrm{mL} \mathrm{m}^{-1}$. As amostras foram analisadas por espectrofotometria na região do ultravioleta em $251 \mathrm{~nm}$. Os valores de recuperação, expressos em porcentagem, foram determinados a partir das respostas analíticas obtidas com os adicionados em função da quantidade teórica de padrão adicionado.

\section{Exatidão (procedimento de dissolução)}

A exatidão do ensaio de dissolução foi determinada pelo método do placebo contaminado. Placebos correspondentes ao processo de manipulação das cápsulas das formulações $\mathrm{A}, \mathrm{B}$ e C, respectivamente, foram submetidos às condições padronizadas para o ensaio de dissolução em triplicata, onde quantidades crescentes de padrão secundário foram adicionadas às cubas para obtenção de três níveis de concentração da curva de calibração: 6,8; 8,4 e 10,8 $\mu \mathrm{g} \mathrm{mL} \mathrm{m}^{-1}$. Após a realização dos ensaios, alíquotas foram coletadas, filtradas em papel de filtro quantitativo e diluídas 10 vezes em meio de dissolução. As amostras foram analisadas por espectrofotometria na região do ultravioleta em $251 \mathrm{~nm}$. Os valores de recuperação, expressos em porcentagem, foram determinados a partir das respostas analíticas obtidas com os adicionados em função da quantidade teórica de padrão adicionado.

\section{Estabilidade em solução}

Para avaliação da estabilidade do padrão de cinarizina em solução de $\mathrm{HCl} 0,1 \mathrm{~mol} \mathrm{~L}^{-1}$, soluções estoque de cinarizina na concentração de $83,33 \mu \mathrm{g} \mathrm{mL}^{-1}$ foram preparadas em triplicata. As soluções foram diluídas imediatamente após o preparo para a concentração final de $8,33 \mu \mathrm{g} \mathrm{mL} \mathrm{m}^{-1}$, utilizando o mesmo solvente, e foram analisadas por espectrofotometria na região do ultravioleta em $251 \mathrm{~nm}$. As soluções estoque foram então mantidas sob refrigeração à $4{ }^{\circ} \mathrm{C}$. Após $24 \mathrm{~h}$, as soluções foram diluídas novamente para a concentração final de $8,33 \mu \mathrm{g} \mathrm{mL} \mathrm{m}^{-1} \mathrm{e}$ analisadas através do método proposto. A média dos valores obtida no tempo 0 foi então comparada com a média obtida no tempo 24 h, utilizando uma análise de variância ao nível de significância de $5 \%$.

Para avaliação da estabilidade de amostras de cinarizina, 6 cápsulas foram submetidas ao ensaio de dissolução sob as condições padronizadas. Alíquotas de $10 \mathrm{~mL}$ foram retiradas após $30 \mathrm{~min}$ de ensaio e imediatamente filtradas. Posteriormente, $1 \mathrm{~mL}$ de cada filtrado foi diluído para o volume de $10 \mathrm{~mL}$ em solução de $\mathrm{HCl} 0,1$ mol L ${ }^{-1}$, obtendo-se soluções na concentração de $8,33 \mathrm{ug} \mathrm{mL}^{-1}$. As soluções foram analisadas imediatamente após o preparo, utilizando o método espectrofotométrico proposto. O restante do filtrado foi mantido à temperatura ambiente por $2 \mathrm{~h}$ e, então, diluído 10 vezes em solução de $\mathrm{HCl}$ 0,1 mol L-1 . As soluções foram analisadas por espectrofotometria em $251 \mathrm{~nm}$. A média dos valores obtida no tempo 0 foi então comparada com a média obtida no tempo $2 \mathrm{~h}$, utilizando uma análise de variância ao nível de significância de 5\%.

\section{Robustez}

A fim de se verificar se o método é robusto a pequenas e deliberadas variações das condições otimizadas, o teste de robustez proposto por Youden e Steiner ${ }^{53}$ foi aplicado. Este teste consiste na análise multivariada de sete variáveis que podem influenciar no resultado analítico. As sete variáveis selecionadas foram: tempo de dissolução, concentração de $\mathrm{HCl}$, velocidade de agitação dos aparatos, temperatura do meio de dissolução, desaeração dos meios de dissolução, filtração das amostras após coleta das amostras e comprimento de onda das leituras. As condições normais das variáveis foram denotadas por letras maiúsculas A, B, C, D, E, F e G e seus valores alternativos foram denotados pelas correspondentes letras minúsculas a, b, c, d, e, f e g. Um total de 8 experimentos foram conduzidos, como indicado 
Tabela 3. Variáveis e seus níveis usadas em estudo de robustez, de acordo com teste de robustez de Youden e Steiner ${ }^{53}$

\begin{tabular}{|c|c|c|c|c|c|c|c|c|c|c|}
\hline \multirow[t]{2}{*}{ Variável } & \multirow{2}{*}{$\begin{array}{c}\text { Condições } \\
\text { nominais }\end{array}$} & \multirow{2}{*}{$\begin{array}{l}\text { Condições } \\
\text { alternativas }\end{array}$} & \multicolumn{8}{|c|}{ Condição experimental } \\
\hline & & & 1 & 2 & 3 & 4 & 5 & 6 & 7 & 8 \\
\hline Tempo de dissolução & $30 \min (\mathrm{A})$ & $35 \min (a)$ & A & A & A & A & $\mathrm{a}$ & $\mathrm{a}$ & $\mathrm{a}$ & a \\
\hline Concentração de $\mathrm{HCl}$ & $0,1 \mathrm{~mol} \mathrm{~L}^{-1}(\mathrm{~B})$ & $0,09 \mathrm{~mol} \mathrm{~L}^{-1}(\mathrm{~b})$ & B & B & $\mathrm{b}$ & $\mathrm{b}$ & B & B & $\mathrm{b}$ & $\mathrm{b}$ \\
\hline Velocidade de agitação & $100 \mathrm{rpm}(\mathrm{C})$ & 95 rpm (c) & $\mathrm{C}$ & $\mathrm{c}$ & $\mathrm{C}$ & $\mathrm{c}$ & $\mathrm{C}$ & $\mathrm{c}$ & $\mathrm{C}$ & $\mathrm{c}$ \\
\hline Temperatura do meio & $37^{\circ} \mathrm{C}(\mathrm{D})$ & $35^{\circ} \mathrm{C}(\mathrm{d})$ & $\mathrm{D}$ & $\mathrm{D}$ & $\mathrm{d}$ & $\mathrm{d}$ & $\mathrm{d}$ & $\mathrm{d}$ & $\mathrm{D}$ & D \\
\hline Desaeração & $\operatorname{sim}(E)$ & não (e) & $\mathrm{E}$ & e & $\mathrm{E}$ & $\mathrm{e}$ & $\mathrm{e}$ & $\mathrm{E}$ & $\mathrm{e}$ & $\mathrm{E}$ \\
\hline Filtro de papel & $\operatorname{sim}(\mathrm{F})$ & não (f) & $\mathrm{F}$ & $\mathrm{f}$ & $\mathrm{f}$ & $\mathrm{F}$ & $\mathrm{F}$ & $\mathrm{f}$ & $\mathrm{f}$ & $\mathrm{F}$ \\
\hline Comprimento de onda & $251 \mathrm{~nm}(\mathrm{G})$ & 254 nm (g) & G & $\mathrm{g}$ & $\mathrm{g}$ & G & $\mathrm{g}$ & G & G & $\mathrm{g}$ \\
\hline Resultados observados & & & $\mathrm{s}$ & $\mathrm{t}$ & $\mathrm{u}$ & $\mathrm{v}$ & w & $\mathrm{x}$ & $\mathrm{y}$ & z \\
\hline
\end{tabular}

Letras maiúsculas representam condições nominais. Letras minúsculas representam condições alternativas

na Tabela 3. A partir desses resultados, o efeito de cada variável foi estimado pela diferença entre a média dos resultados das 4 análises com letra maiúscula e a média dos resultados das 4 análises com letra minúscula. Considerando o desvio padrão entre os 8 resultados, os valores de efeito maior do que o desvio padrão multiplicado pela raiz quadrada de dois $(\mathrm{s} \sqrt{2}$ ) foram considerados significantes e, portanto, alteram a resposta a resposta analítica.

\section{Limite de detecção $(L D)$ e limite de quantificação $(L Q)$}

O LD e o LQ das amostras (A, B e C), sob as condições experimentais estabelecidas, foram determinados efetuando-se 10 leituras correspondentes ao branco (solução de HCL $0,1 \mathrm{~mol} \mathrm{~L}^{-1}$ ). As estimativas do LD e do LQ foram então determinadas através das seguintes equações: $\mathrm{LD}=(\mathrm{DP} \times 3) / \mathrm{IC}$ e LQ $=(\mathrm{DP} \times 10) / \mathrm{IC}$, em que DP é o desvio padrão relativo entre as leituras do branco e IC é a inclinação da curva analítica obtida no estudo de linearidade.

\section{Cinética de dissolução e eficiência de dissolução (ED\%)}

Para o estudo da cinética de dissolução, foram aplicados os modelos de ordem zero, plotando-se os valores da quantidade de fármaco não dissolvido versus tempo, e de primeira ordem, através de gráficos do logaritmo neperiano (In) da quantidade de fármaco não dissolvido versus tempo. Na construção dos modelos, foram considerados apenas os pontos ascendentes do perfil de dissolução. Os parâmetros cinéticos da dissolução, como a constante de velocidade de dissolução $(K)$ e a meia-vida de dissolução $\left(\mathrm{t}_{50 \%}\right)$, foram determinados a partir das equações definidas pelo modelo matemático que apresentou o melhor e mais significativo índice de correlação.

A eficiência de dissolução (ED\%) foi calculada a partir da razão entre a área sob a curva de dissolução (ASC) da cinarizina no intervalo de tempo compreendido entre 0 e 60 min e a área total do retângulo (ATR) definido pela ordenada (100\% de dissolução) e pela abscissa (tempo igual a $60 \mathrm{~min}$ ).

\section{RESULTADOS E DISCUSSÃO}

\section{Estabelecimento de condições de ensaio de dissolução}

Os valores de solubilidade da cinarizina a $37^{\circ} \mathrm{C}$ em diferentes valores de $\mathrm{pH}$ foram descritos por $\mathrm{Gu}$ e colaboradore ${ }^{46}$ e estão reproduzidos a seguir: $290 \mu \mathrm{g} \mathrm{mL} \mathrm{em}^{-1} \mathrm{em}$ solução de $\mathrm{HCl} 0,1 \mathrm{~mol} \mathrm{~L}^{-1}, \mathrm{pH}$ 2.0; $17 \mu \mathrm{g} \mathrm{mL}^{-1}$ em pH 5,0; 2,0 $\mu \mathrm{g} \mathrm{mL}^{-1}$ em pH 6,5 e 3,0 $\mu \mathrm{g} \mathrm{mL}^{-1}$ em solução-tampão $\mathrm{pH} 6,5 .{ }^{46} \mathrm{~A}$ cinarizina, portanto, é uma base fraca com baixa solubilidade em solventes aquosos e sua solubilidade é altamente dependente do $\mathrm{pH}$. Dessa forma, estabeleceu-se como meio ideal para realização de ensaios de dissolução solução de $\mathrm{HCl} 0,1 \mathrm{~mol} \mathrm{~L}^{-1}$,

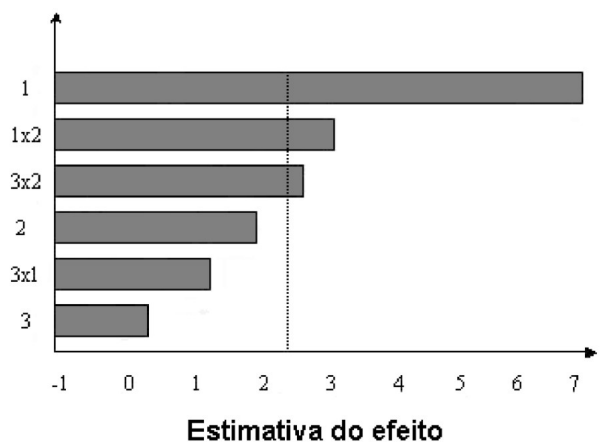

Figura 2. Diagrama de Pareto (1: velocidade, 2: tempo, 3: aparato)

a fim de se assegurar uma máxima solubilidade do fármaco, pois a solução de $\mathrm{HCl} 0,1 \mathrm{~mol} \mathrm{~L}^{-1}$ é o meio que possui o menor $\mathrm{pH}$ dentre os meios normalmente utilizados em ensaios de dissolução. ${ }^{1}$ Outros meios como solução-tampão acetato $\mathrm{pH} 4,5$, solução-tampão fosfato pH 6,8 e água não foram testados, pois não possuem uma solubilidade adequada e não asseguram, portanto, as condições sink que são recomendadas para realização de ensaios de dissolução. ${ }^{1}$

Após definição de solução de $\mathrm{HCl} 0,1 \mathrm{~mol} \mathrm{~L}^{-1}$ como meio de dissolução, realizou-se o planejamento fatorial, utilizando diferentes aparatos, tempos e velocidades de agitação como representado nas Tabelas 1 e 2. A partir dos resultados obtidos, construiu-se o Diagrama de Pareto, apresentado na Figura 2.

A visualização do diagrama nos permite inferir que o fator velocidade de agitação é estatisticamente significativo, sendo o efeito deste fator positivo, o que nos indica que o maior nível, dentro do domínio experimental, produz uma maior liberação de cinarizina da forma farmacêutica cápsula. Sendo assim, a fim de se obter uma maior liberação desse fármaco que apresenta baixa solubilidade em solventes aquosos, a velocidade de agitação foi fixada em $100 \mathrm{rpm}$. O fator tempo não foi significativo, pois a estimativa do seu efeito foi menor do que o erro (Figura 2), o que significa que a quantidade de fármaco liberada em 30 min não difere da quantidade liberada em $60 \mathrm{~min}$. Portanto, o tempo de 30 min foi fixado, levando em consideração a velocidade de análise, que é um fator de extrema importância em laboratórios de controle de qualidade. A análise da Figura 2 também nos permite concluir que o efeito do tipo de aparato não é significativo. Considerando que a farmacopeia americana recomenda cesta para análise de cápsulas, ${ }^{54}$ esse aparato foi selecionado como ideal para realização de ensaios de dissolução de cápsulas de cinarizina. Portanto, as condições otimizadas com base nos resultados obtidos foram: solução de $\mathrm{HCl} 0,1 \mathrm{~mol} \mathrm{~L}^{-1}$ $\left(37 \pm 0,5^{\circ} \mathrm{C}\right)$ como meio de dissolução, aparato cesta, velocidade de agitação $100 \mathrm{rpm}$ e tempo de $30 \mathrm{~min}$ para coleta das amostras. 


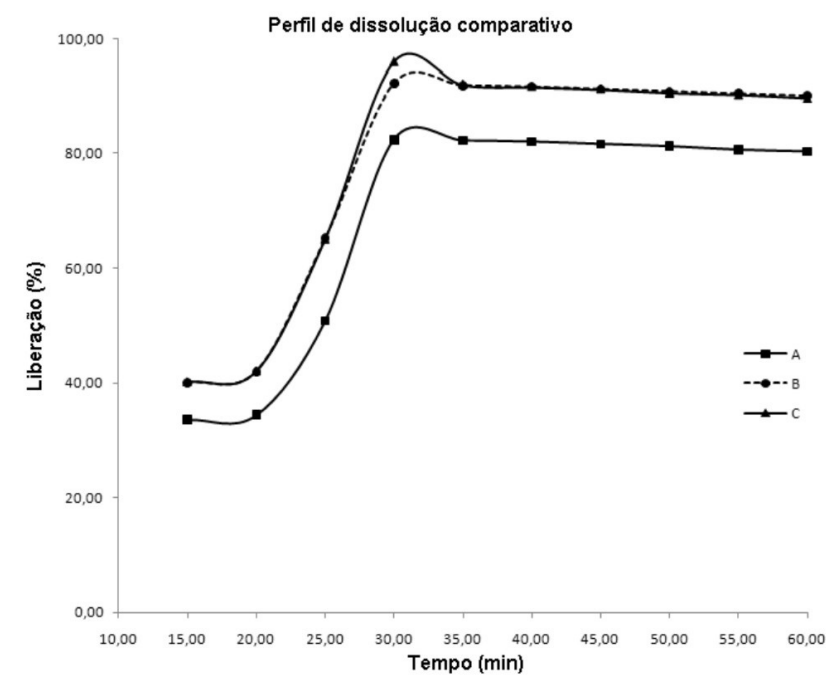

Figura 3. Perfis de dissolução das formulações $A, B$ e C, utilizando solução de $\mathrm{HCl} 0,1 \mathrm{~mol} \mathrm{~L}^{-1}$ como meio dissolução $\left(37 \pm 0,5^{\circ} \mathrm{C}\right)$, cesta como aparato, velocidade de agitação de 100 rpm e quantificação por espectrofotometria em $251 \mathrm{~nm}$

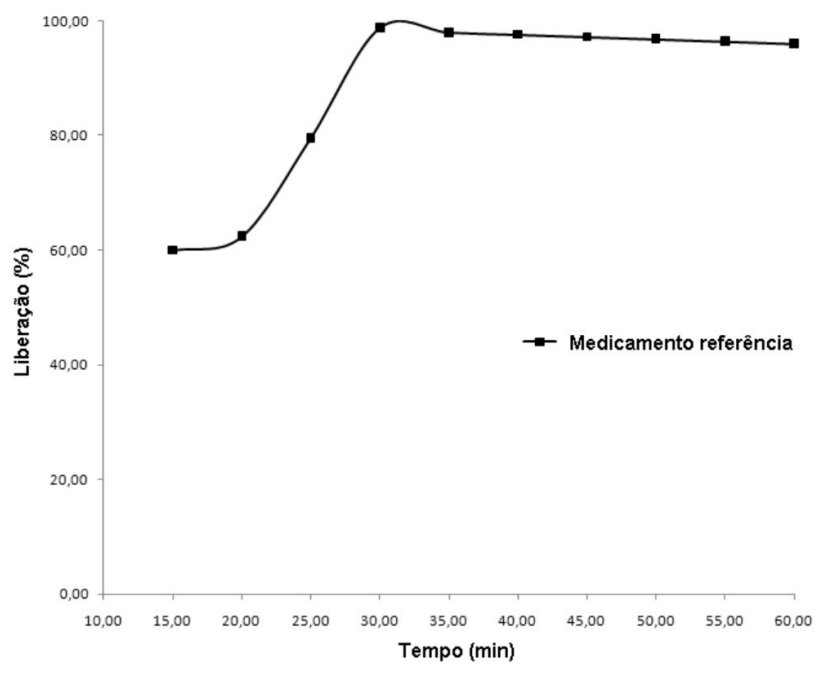

Figura 4. Perfil de dissolução de cinarizina medicamento de referência, utilizando solução de $\mathrm{HCl} 0,1 \mathrm{~mol} \mathrm{~L}^{-1}$ como meio dissolução $\left(37 \pm 0,5{ }^{\circ} \mathrm{C}\right)$, cesta como aparato, velocidade de agitação de $100 \mathrm{rpm}$ e quantificação por espectrofotometria em $251 \mathrm{~nm}$

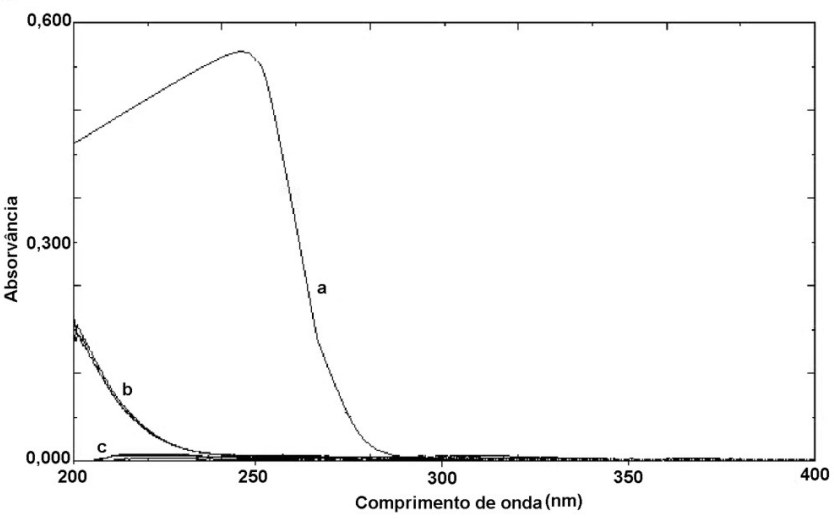

Figura 5. Sobreposição de espectros de cinarizina solução padrão a $8,33 \mu \mathrm{g}$ $m L^{-1}(a)$, cápsulas vazias $A, B$ e $C(b)$, e excipientes $A, B$ e $C(c)$ na determinação da seletividade do método para cinarizina, utilizando como solvente solução de $\mathrm{HCl}$ 0,1 $\mathrm{mol} \mathrm{L}^{-1}$
Após definição de condições ideais para realização de ensaios de dissolução de cápsulas de cinarizina, construíram-se perfis de dissolução de três produtos manipulados em diferentes farmácias magistrais, A, B e C, respectivamente. A observação da Figura 3 nos leva à conclusão de que os medicamentos $\mathrm{B}$ e $\mathrm{C}$ apresentaram percentuais de liberação do fármaco acima de $85 \%$ em 30 min do ensaio. A visualização dos perfis também nos permite concluir que após o tempo de 30 min a liberação do fármaco a partir dos três produtos não se altera significativamente, confirmando que o tempo de 30 min é ideal como único ponto para realização de teste de dissolução em laboratórios de análise de rotina. Com bases nesses resultados, o critério de aceitação de $85 \%$ foi estabelecido para avaliação da qualidade de cápsulas contendo $75 \mathrm{mg}$ de cinarizina, o que é normalmente estabelecido para fármacos de liberação imediata. ${ }^{54}$ Para o medicamento A, entretanto, o percentual de liberação foi abaixo de $85 \%$, o que indica que este produto está abaixo da especificação, provavelmente devido ao processo de manipulação ou à composição de excipientes presentes nesta formulação.

O medicamento referência contendo cinarizina $75 \mathrm{mg}$ Stugeron $^{\circledR}$ foi submetido às mesmas condições do ensaio de dissolução estabelecido para a forma farmacêutica cápsula, objetivando-se a reprodução das condições otimizadas. O ensaio de dissolução para os comprimidos demonstrou resultados satisfatórios para o medicamento referência que apresentou liberação acima de 85\% (próximo a 100\%) após 30 min de ensaio, como demonstrado na Figura 4. Além disso, pode-se observar nessa figura que após 30 min o perfil de dissolução do medicamento referência permanece praticamente estável.

\section{Validação}

A curva analítica para cinarizina padrão secundário mostrou linearidade adequada na faixa de 6,8 a $10,8 \mu \mathrm{g} \mathrm{mL}^{-1}$, com r $=0,9992$ (coeficiente de correlação). A equação da reta obtida foi: $y=0,0382 x+$ 0,1357. A seletividade, verificada com o método espectrofotométrico, demonstrou não haver interferência dos excipientes no comprimento de onda de $251 \mathrm{~nm}$ (Figura 5).

Os resultados de precisão do método analítico e do ensaio de dissolução estão demonstrados na Tabela 4. Os valores de estimativa de desvio padrão (DPR) inferiores a 5\% intradia e interdia, considerando-se o procedimento de dissolução e o método de análise, indicam precisão satisfatória.

Tabela 4. Precisão de método analítico e ensaio de dissolução para cápsulas de cinarizina $75 \mathrm{mg}$

\begin{tabular}{cccc}
\hline Parâmetro & Produto & $\begin{array}{c}\text { Média das } \\
\text { absorvâncias }\end{array}$ & $\begin{array}{c}\text { D.P.R. } \\
(\%)\end{array}$ \\
\hline $\begin{array}{c}\text { Precisão intradia do } \\
\text { método de análise }\end{array}$ & Padrão secundário & 0,454 & $0,22(\mathrm{n}=6)$ \\
$\begin{array}{c}\text { Precisão interdia do } \\
\text { método de análise }\end{array}$ & Padrão secundário & 0,454 & $1,54(\mathrm{n}=12)$ \\
Precisão intradia do & A & 0,377 & $1,58(\mathrm{n}=6)$ \\
ensaio de dissolução & $\mathrm{B}$ & 0,419 & $2,15(\mathrm{n}=6)$ \\
& $\mathrm{C}$ & 0,449 & $0,54(\mathrm{n}=6)$ \\
Precisão interdia do & $\mathrm{A}$ & 0,375 & $1,46(\mathrm{n}=12)$ \\
ensaio de dissolução & $\mathrm{B}$ & 0,419 & $2,34(\mathrm{n}=12)$ \\
& $\mathrm{C}$ & 0,447 & $1,37(\mathrm{n}=12)$ \\
\hline
\end{tabular}

D.P.R. = Estimativa de desvio padrão relativo entre as determinações.

$\mathrm{Na}$ avaliação da exatidão, valores de recuperação entre 97,7 a 99,4\% para o método de análise e entre 96,9 a 99,6\% para o procedimento de dissolução indicam exatidão adequada (Tabela 5). De acordo com a literatura, valores entre $95,0-105,0 \%$ para ensaios de dissolução são considerados satisfatórios. ${ }^{55}$ 
Tabela 5. Exatidão de método analítico e ensaio de dissolução para cápsulas de cinarizina $75 \mathrm{mg}$

\begin{tabular}{cccc}
\hline Parâmetro & Produto & Recuperação (\%) & D.P.R. (\%) \\
\hline Exatidão do método & $\mathrm{A}$ & 97,7 & 0,22 \\
de análise & $\mathrm{B}$ & 98,2 & 0,26 \\
& $\mathrm{C}$ & 99,4 & 0,45 \\
Exatidão do ensaio & $\mathrm{A}$ & 96,9 & 0,41 \\
de dissolução & $\mathrm{B}$ & 98,7 & 0,23 \\
& $\mathrm{C}$ & 99,6 & 0,27
\end{tabular}

D.P.R. = Estimativa de desvio padrão relativo entre as determinações.

Os ensaios de estabilidade em solução mostraram que o padrão de cinarizina permaneceu estável quando mantido em solução de $\mathrm{HCl}$ 0,1 mol L ${ }^{-1}$ à $4{ }^{\circ} \mathrm{C}$ por $24 \mathrm{~h}$, pois a análise de variância ao nível de significância de $5 \%$ mostrou que não houve diferenças estatísticas entre a média das determinações no tempo 0 e a média das determinações no tempo $24 \mathrm{~h}\left(\mathrm{~F}_{\text {calculado }}=5,83\right.$ e $\left.\mathrm{F}_{\text {crítico }}=18,51\right)$. Além disso, as amostras permaneceram estáveis por $2 \mathrm{~h}$ à temperatura ambiente, após terem sido submetidas ao ensaio de dissolução sob as condições padronizadas, pois a análise de variância ao nível de significância de $5 \%$ mostrou que não houve diferenças estatísticas entre a média das determinações no tempo 0 e a média das determinações no tempo 2 $\mathrm{h}\left(\mathrm{F}_{\text {calculado }}=0,74\right.$ e $\left.\mathrm{F}_{\text {crítico }}=4,96\right)$.

Os resultados das 8 análises realizadas no ensaio de robustez foram utilizados para calcular o efeito de cada variável (diferença entre a média dos resultados das 4 análises com letra maiúscula e a média dos resultados das 4 análises com letra minúscula), bem como o desvio padrão entre os 8 resultados. Valores de efeito maior do que o desvio padrão multiplicado pela raiz quadrada de dois $(\mathrm{s} \sqrt{2})$ foram considerados significantes e, portanto, alteram a resposta a resposta analítica. De acordo com a observação da Tabela 6, pode-se concluir que nenhum efeito foi maior do que o critério $\sqrt{ } \sqrt{2}$, o que significa que nenhum fator testado influencia significativamente na resposta analítica. A partir desses resultados, podese deduzir que o método é considerado robusto para todas as variáveis consideradas dentro do domínio experimental considerado.

Tabela 6. Valores de efeitos obtidos de acordo com o teste de Youden e Steiner ${ }^{53}$

\begin{tabular}{cc}
\hline Variável & Efeito \\
\hline Tempo de dissolução & 0,00 \\
pH do meio de dissolução & 0,01 \\
Velocidade de agitação & 0,01 \\
Temperatura do meio & $-0,02$ \\
Desaeração & 0,01 \\
Filtro de papel & $-0,01$ \\
Exposição à luz & $-0,02$ \\
D.P. x $\sqrt{2} *$ & 0,03 \\
\hline
\end{tabular}

*D.P. $x \sqrt{ } 2=$ Desvio padrão entre os resultados das 8 combinações experimentais (Tabela 3 ) multiplicado pela raiz quadrada de 2.

Os limites de detecção e de quantificação foram respectivamente de 0,076 e $0,253 \mu \mathrm{g} \mathrm{mL}^{-1}$, mostrando que todas as análises foram realizadas em níveis de concentrações acima desses limites.

\section{Cinética de dissolução e eficiência de dissolução (ED\%)}

Foi realizado o estudo da cinética do processo de dissolução, empregando-se os modelos de ordem zero e primeira ordem. Através da linearização das curvas de dissolução, foram obtidos os respectivos coeficientes de correlação (r). O modelo considerado mais adequado, ou seja, o que apresentou melhor correlação foi o de ordem zero para todos os produtos (Tabela 7).

Tabela 7. Parâmetros estatísticos dos estudos de regressão aplicando modelo de ordem zero e primeira ordem, derivados dos perfis de dissolução dos produtos A, B, C e referência após o ensaio de dissolução sob condições otimizadas

\begin{tabular}{ccc}
\hline Produto & $\begin{array}{c}\text { Ordem zero } \\
(\mathrm{r})\end{array}$ & $\begin{array}{c}\text { Primeira ordem } \\
(\mathrm{r})\end{array}$ \\
\hline A & 0,9729 & 0,8839 \\
B & 0,9722 & 0,8333 \\
C & 0,9656 & 0,7852 \\
Referência & 0,9889 & 0,8164 \\
\hline
\end{tabular}

A partir do modelo de ordem zero, foram determinados os parâmetros cinéticos da constante de velocidade de dissolução (k) e da meiavida de dissolução $\left(\mathrm{t}_{50 \%}\right)$. Os resultados indicaram perfis de dissolução diferenciados, e os valores da meia-vida de dissolução $\left(\mathrm{t}_{50 \%}\right)$ apresentados apontam bom desempenho de liberação para os produtos analisados (Tabela 8). A eficiência de dissolução (ED\%), também demonstrada na Tabela 8, nos permite observar que o produto B apresentou perfil mais próximo do perfil do produto referência. Uma análise estatística, que normalmente é recomendada para a comparação de perfis, não foi utilizada, pois a forma farmacêutica do produto referência (comprimido) testado é diferente da forma farmacêutica das amostras (cápsulas), não sendo aplicável, portanto, a comparação entre perfis.

Tabela 8. Valores da constante de velocidade de dissolução (k), meia-vida de dissolução $\left(\mathrm{t}_{50 \%}\right)$ e eficiência de dissolução (ED\%) para cinarizina produtos A, B, C e referência

\begin{tabular}{cccc}
\hline Produto & $\mathrm{t}_{50 \%}(\mathrm{~min})$ & $\mathrm{k}\left(\mathrm{min}^{-1}\right)$ & $(\mathrm{ED} \%)$ \\
\hline $\mathrm{A}$ & 21,08 & 2,53 & 57,53 \\
$\mathrm{~B}$ & 18,74 & 2,88 & 65,02 \\
C & 18,49 & 2,96 & 65,32 \\
Referência & 14,83 & 3,18 & 74,12 \\
\hline
\end{tabular}

Uma consideração importante sobre o trabalho é que cápsulas de algumas farmácias de manipulação não apresentaram dissolução significativa em solução de $\mathrm{HCl} 0,1 \mathrm{~mol} \mathrm{~L}^{-1}$. Isto foi verificado provavelmente devido à ausência de lauril sulfato de sódio nestas formulações, pois se sabe que o lauril sulfato de sódio age como tensoativo, aumentando a velocidade de dissolução de sólidos.$^{56}$ Estas cápsulas foram desconsideradas neste estudo, pois não asseguram uma liberação efetiva, como foi observado com o medicamento referência.

\section{CONCLUSÕES}

As melhores condições para realização de ensaio de dissolução de cápsulas de cinarizina $75 \mathrm{mg}$ são: solução de $\mathrm{HCl} \mathrm{0,1} \mathrm{mol} \mathrm{L}^{-1}(900 \mathrm{~mL}$, a $37 \pm 0,5^{\circ} \mathrm{C}$ ) como meio de dissolução, aparato cesta, velocidade de agitação de 100 rpm e tempo de coleta em 30 min. O ensaio de dissolução proposto mostrou seletividade, linearidade, precisão, robustez, exatidão e adequados limites de detecção e de quantificação. Portanto, é adequado para ensaios de rotina em laboratórios de controle de qualidade.

\section{AGRADECIMENTOS}

Às farmácias de manipulação que forneceram os medicamentos A, B e C, respectivamente, à FAPEMIG pela bolsa de IC e ao Centro 
de Equivalência Farmacêutica do Núcleo de Controle de Qualidade da Universidade Federal de Alfenas.

\section{REFERÊNCIAS}

1. United States Food and Drug Administration, Center for Drug Evaluation and Research (CDER); Dissolution Testing of Immediate Release Solid Oral Dosage Forms. Guidance for Industry, Rockville, 1997.

2. Dalmora, S. L.; Nogueira, D. R.; Calegari G. Z.; Bergamo, A. C.; Stamm, F. P.; Quim. Nova 2010, 33, 1150.

3. Vaucher, L. C.; Paim, C. S.; Lange, A. D.; Schapoval, E. E. S.; Quim. Nova 2009, 32, 1329.

4. Soares, M. F. de La R.; Soares Sobrinho, J. L.; Granjeiro Jr, S.; da Silva, K. E. R.; Alves, L. D. S.; Rolim Neto, P, J.; Quim. Nova 2010, 33, 478.

5. García-Ruiz, P. J.; Jiménez-Jiménez, F. J.; Yébenes, J. G.; Parkinsonism Relat. Disord. 1998, 4, 211.

6. Korolkovas, A.; Dicionário Terapêutico Guanabara, ed. 2006/2007, Guanabara Koogan: Rio de Janeiro, 2007.

7. Teive, H. A. G.; Troiano, A. R.; Germiniani, F. M. B.; Werneck, L. C.; Parkinsonism Relat. Disord. 2004, 10, 243.

8. Abdelal, A. A.; Kitagawa, S.; Ohtani, H.; El-Enany, N.; Belal, F.; Walash M. I.; J. Pharm. Biomed. Anal. 2008, 46, 491 .

9. Mangelings, D.; Discry, J.; Maftouh, M.; Massart, D. L.; Heyden, Y. V.; Electrophoresis 2005, 26, 3930

10. Xie, X. T.; Wu, G. L.; Liu, C. H.; Sери 1993, 11, 315.

11. Woestenborghs, R.; Michielsen, L.; Lorreyne, W., Heykants, J.; J. Chromatogr., B: Anal. Techno. Biomed. Life Sci. 1982, 232, 85.

12. Sane, R. T.; Sahasrabudhe, S. P.; Nayak, V. G.; Ladage, K. D.; Kothurkar, R. M.; Nayak, V. G.; Indian Drugs 1989, 26, 491.

13. Zarapkar, S. S.; Bhandari, N. P.; Halker, U. P.; Indian Drugs 2000, 37, 295.

14. Argekar, A. P.; Shah, S. J.; J. Pharm. Biomed. Anal. 1999, 19, 813.

15. Bardarov, V.; Zikolova, T.; Radoevska, N.; Sahtura, A.; Pharmacia 2008, 55,14

16. El-Gindy, A.; Emara, S.; Hadad. G. M.; Il Farmaco 2004, 59, 703.

17. El-Gindy, A.; Emara, S.; Mostafa, A.; Il Farmaco 2004, 59, 713.

18. Hassan, S. S. M.; Elmosallamy, M. A. F.; Abbas, A. B.; J. Pharm. Biomed. Anal. 2002, 28, 711.

19. Metwally, F. H.; Elzeany, B. A.; Darwish, H. W.; J. AOAC Int. 2005, 88, 1666.

20. Argekar, A. P.; Powar, S. G.; J. Planar Chromatogr. Mod. TLC 1999, 12, 272.

21. Bagade, S. B.; Walode, S. G.; Charde, M. S.; Tajne, M. R.; Kasture, A. V.; Asian J. Chem. 2005, 17, 1116.

22. Boneva, A. S.; Loginova, N. F.; Mishchenko, V. V.; Starostina, A. K.; Torosyan, Z. K.; Nino, N. S.; Mairanovskii, V. G.; Khim. Farm. Zh. 1983, 17, 1133.

23. Zeng, Y. H.; Sun, H. Y.; Fenxi Huaxue 1993, 21, 1185.

24. El-Sayed, G. O.; Yasin, S. A.; El Badawy, A. A.; Anal. Lett. 2008, 41, 3021.

25. Hegde, R. N.; Hosamani, R. R.; Nandibewoor, S. T.; Colloids Surf., B 2009, 72, 259 .

26. Hassan, S. S. M.; Abbas, A. B.; Elmosallamy, M. A. E.; Mikrochim. Acta 1998, 128, 69 .

27. Abdine, H.; Belal, F.; Zoman, N.; Il Farmaco 2002, 57, 267.

28. Abdel-Khalek, M. M.; Abdel-Hamid, M. E.; Mahrous, M. S.; AbdelSalam, M. A.; Anal. Lett. 1985, 18, 781.
29. Saleh, G. A.; Askal, H. F.; Pharmazie 1990, 45, 220.

30. Patil, S. B.; Nemade, S. P.; Chaudhari, G. N.; Kolte, H. V.; Indian Drugs 1993, 30, 438 .

31. Zhao, F. L.; Xu, B. Z.; Zhang, Z. Q.; Tong, S. Y.; J. Pharm. Biomed. Anal. 1999, 21, 355.

32. Daabees, H. G.; Spectrosc. Lett. 1999, 32, 913.

33. Tokumura, T.; Ichikawa, T.; Sugawara, N.; Tatsuishi, K.; Kayano, M.; Machida, Y.; Hoshida, H.; Nagai, T.; Chem. Pharm. Bull. 1985, 33, 2069.

34. Devagondanahalli, M. H.; Shaikh, S. M. T.; Jaldappagari, S.; Ramanaboyina, S. K.; Kasalanti, H.; J. Chin. Chem. Soc. 2007, 54, 63.

35. El-Bardicy, M. G.; Lotfy, H. M.; El-Sayed, M. A.; El-Tarras, M. F.; J. AOAC Int. 2008, 91, 299.

36. Vinodhini, C.; Vaidhyalingam, V.; Ajithadas, A.; Niramathi, A.; Shantha, A.; Indian Drugs 2002, 39, 491.

37. Salem, M. Y.; El-Zanfaly, E. S.; El-Tarras, M. F.; El-Bardicy, M. G.; Anal. Bioanal. Chem. 2003, 375, 211.

38. Salem, M. Y.; El-Bardicy, M. G.; El-Tarras, M. F.; El-Zanfally, E. S.; J. Pharm. Biomed. Anal. 2002, 30, 21.

39. Neagu, A. F.; Constantinescu, I. C.; Nedelcu, A.; Florea, M.; Farmacia 2008, 56,42 .

40. Salem, H.; Chin. Pharm. J. 1999, 51, 123.

41. Xu, B.; Zhao, F.; Tong, S.; Guang Pu Xue Yu Guang Pu Fen Xi/Spectrosc. Spectral Analysis 1999, 19, 887.

42. Townshend, A.; Youngvises, N.; Wheatley, R. A.; Liawruangrath, S.; Anal. Chim. Acta 2003, 499, 223.

43. Walash, M. I.; Belal, F.; El-Enany, N.; Abdelal, A. A.; J. AOAC Int. 2008, 91, 349 .

44. Walash, M. I.; Belal, F.; El-Enany, N.; Abdelal, A. A.; J. Fluoresc. 2008, 18,61 .

45. Peeters, K.; de Maesschalck, R.; Bohets, H.; Vanhoutte, K.; Nagels, L.; Eur. J. Pharm. Sci. 2008, 34, 243.

46. Gu, C.-H.; Rao, D.; Gandhi, R. B.; Hilden, J.; Raghavan, K.; Eur. J. Pharm. Sci. 2005, 94, 199.

47. Bonfilio, R.; Mendonça, T. F.; Pereira, G. R.; de Araújo, M. B.; Tarley, C. R. T.; Quim. Nova 2010, 33, 377.

48. Montgomery, D. C. Em Design and Analysis of Experiments; Montgomery, D. C., ed.; Wiley: New York, 2000, p. 177-185.

49. Brasil, Agência Nacional de Vigilância Sanitária; Resolução RE nº89, 29/5/2003, Guia para validação de métodos analíticos e bioanalíticos, Diário Oficial da União, Brasília, 02 jun. 2003 - http://www.anvisa.gov. br, acessada em Julho 2010.

50. Association of Official Analytical Chemists; Official methods of analysis of $A O A C, 16^{\text {th }}$ ed., AOAC International: Arlington, 1995.

51. ICH Q2(R1), International Conference on Harmonization; Validation of analytical procedures: Text and methodology, 2005 - http://www.ich.org/ LOB/media/ MEDIA417.pdf , acessada em Fevereiro 2010.

52. Ribani, M.; Bottoli, C. B. G.; Collins, C. H.; Jardim, I. C. S. F.; Melo, L. F. C.; Quim. Nova 2004, 27, 771.

53. Youden, W. J.; Steiner E. H.; Statistical Manual of the AOAC, $48^{\text {th }}$ ed., AOAC International: Arlington, 1975.

54. The United States Pharmacopeia, $31^{\text {th }}$ ed., United States Pharmacopoeial Convention: Rockville, 2008.

55. Marques, M. R. C.; Brown, W.; Rev. Anal. 2002, 1, 48.

56. Ruela, A. L. M.; Araújo, M. B.; Pereira, G. R.; Quim. Nova 2009, 32, 165 . 